\title{
STERILE NEUTRINOS AND THE MILGROM'S LAW PROBLEM
}

\author{
Luis ACEDO** \\ Instituto de Matemática Multidisciplinar, \\ Universitat Politècnica de València, \\ Camino de Vera s/n, 46022 Valencia, Spain. E-mail: Luiacrodeimm.upv.es
}

In 1983 Milgrom proposed an alternative explanation for the anomalous rotation of galaxies based upon a modification of Newtonian dynamics for small accelerations (MOND theory) [四]. A careful analysis of the rotation curves revealed that the effect of the supposed dark matter seems to activate only when the orbital acceleration is smaller than $2 \times 10^{-8} \mathrm{~cm} \mathrm{~s}^{-2}$. MOND theory still challenges the Dark Matter community because simulations of cold dark matter (CDM) accretion favour the formation of halos with a cusp at the center of the galaxies (Via Lactea II [ब]]). The calculations of galactic density profiles from the rotation curves contradict the prediction of a halo with a peak at the center of galaxies. This phenomenon is related with Milgrom's law because a galactic core almost free from DM will imply that there exists a critical orbit separating the inner regions of the galaxy, where rotation behaviour can be deduced from the observed mass, and the outer regions where DM must be invoked to explain the discrepancy. We show that this problem is overcome in a Warm Dark Matter theory for sterile neutrinos with a mass around $3 \mathrm{keV}$ [3]]. These particle behave as collisionless Vlasov particles with a primordial typical velocity around $330 \mathrm{~km} \mathrm{~s}^{-1}$ and, consequently, they evaporated from galactic cores and reorganized in halos with a cusp at a finite distance from the galactic center (in contrast with Cold Dark Matter simulations which predict a cusp at the center of galaxies).

Identification of Dark Matter 2010-IDM2010

July 26-30, 2010

Montpellier France

\footnotetext{
* Speaker.

${ }^{\dagger}$ Supported by Research Grant GV/2009/032 from the Generalitat Valenciana and PAID-06-09 (ref: 2588) from the Universitat Politècnica de València .
} 


\section{Introduction}

If galaxies were composed only of the observed luminous matter we will expect a decrease of the orbital velocity $\propto r^{-1 / 2}, r$ being the distance to the galactic centre. Nevertheless, a flat asymptotic region in the rotation curve corresponding to a constant orbital velocity of stars and gas clouds is found, $V_{\infty}$. This is the original observation of Fritz Zwicky [四] and the precursor of the current versions of the Dark Matter Hypothesis. Another landmark in the history of the DM hypothesis is marked by the detailed analysis of rotation curves of galaxies published by Faber \& Gallagher [5] in 1979 and Vera Rubin et al. [6] in 1980. After this moment the community of researchers both in astrophysics and physics working to elucidate the nature of this elusive kind of matter is growing steadily.

Nevertheless, a controversy on the DM problem arised only three years after the publication of the careful observations of Faber \& Gallagher and Rubin and her collaborators. M. Milgrom suggested that a modification of Newtonian dynamics for small accelerations (MOND theory) [W] was necessary in order to explain that the effect of DM only activates for orbital accelerations smaller than $a_{0} \approx 2 \times 10^{-8} \mathrm{~cm} \mathrm{~s}^{-2}$. Milgrom assumed that the equivalence principle of general relativity is not valid for small accelerations because the mass of an object is a function $m\left(a / a_{0}\right)$, with $a_{0}$ being a new universal constant. From his theory, Milgrom derived an expression for the asymptotic orbital velocity in the galaxies:

$$
V_{\infty}^{4}=a_{0} G M
$$

where $M$ is the observable mass of the galaxy. So, Milgrom explained the key features of the anomalous rotation curves of galaxies without invoking DM and instead proposed a new theory of gravitation as the alternative explanation.

Simulations of cold dark matter (CDM) accretion favour the formation of halos with a cusp at the center of the galaxies (the latest and more precise CDM's halos simulation is known as Via Lactea II [D] ) and this is known since the work of Navarro et al. [G]. The calculations of galactic density profiles from the rotation curves [ [ [ ] and many observations of these rotation curves from galaxies [9] contradict the prediction of a halo with a peak at the center of galaxies. This phenomenon is related with Milgrom's law because a galactic core almost free from DM will imply that there exists a critical orbit separating the inner regions of the galaxy, where rotation behaviour can be deduced from the observed mass, and the outer regions where DM must be invoked to explain the discrepancy. There is an increasing and independent evidence on dark matter (such as Gravitational lensing [ㅁ] by galaxy clusters or Cosmic Microwave Background anisotropies [U], [2]). Consequently, the clarification of Milgrom's paradox, in the context of dark matter models, is urgent. Trying to reconcile DM models with Milgrom's law could also help us in clarifying the properties of the fundamental particles which form it.

Only recently the DM community has picked up the gauntlet thrown by Milgrom's theory and explanations on the absence of halo cusp near the galactic centers have been proposed. Dekel et al.[ए3] proposed that central cusps could have evolved into flattened cores as a consequence of a wave of star formation. Nowadays this is the standard theory for the origin of the flattened cores in the inferred distributions for CDM. However, the stability of the resulting distribution is not clear 
and the possible accretion of particles in the halo to the core of the galaxy after the wave of star formation cannot be excluded.

Browsing through the communications to this conference reveals that Weakly Interacting Massive Particles (WIMPs) are still the preferred candidate for a Dark Matter particle. The CDM scenario provided by WIMPs suffers from the fundamental problem on structure formation pointed out by Milgrom's work. Extensions of the Standard Model have predicted several candidates for a DM particle:

- Axions (rest mass $\mu \sim 10^{-5} \mathrm{eV}$ ).

- Magnetic monopoles $\left(\mu \sim 10^{16} \mathrm{GeV}\right)$.

- Weakly interacting massive particles (WIMPs).

- Neutralinos (masses in the range $\mu \sim 1-1000 \mathrm{GeV}$ ).

- Several Species of Sterile Neutrinos.

The sterile neutrino is a proposal for a family of particles pioneered by Dodelson and Widrow [II]] which considered a model for their production in the early Universe by oscillations. In this case, the predicted mass is around $0.1 \mathrm{keV}$ (very small for allowing the collapse of matter in the protogalaxies). Another model was proposed by Shi and Fuller [प5]] (lepton-number-driven resonant conversion of active neutrinos at the big bang nucleosynthesis epoch). The masses of the resonant sterile neutrinos could be as larger as $10 \mathrm{keV}$ which it is a more reasonable value for preventing "free streaming" and allows the condensation around primordial inhomogeneities. In another scenario, sterile neutrinos are the result of the decay of a heavy scalar [ए6]. In this case there is a chance to detect such a scalar at the Large Hadron Collider. In connection with the heavy scalar scenario, it has also been suggested that some species of sterile neutrinos could also decay into ordinary neutrinos and $\mathrm{X}$ rays that would boost reionization in the early Universe. This will overcome the problem of the large free-streaming length for WDM in the context of structure formation [ए]].

Sterile neutrino interact very weakly with ordinary matter and their direct detection is even more difficult than that of the WIMPs. Inspired by these works we propose a WDM model for Vlasov particles trapped in the combined gravitational field of visible matter and DM. If the velocities of these sterile neutrinos are sufficiently large, they tend to evaporate from the galactic center and a stable halo with a cusp at a distance from the center is formed. The objective of this paper is to show that the core-cusp anomaly that inspired Milgrom's work on an alternative theory of gravitation could be explained in the context of a WDM model with the sterile neutrino as the preferred candidate. This work is organized as follows: In Section $\square$ we discuss the self-gravitating model for sterile neutrinos and a baryonic core and fit the results to the inferred mass distributions for the Milky Way. In Section [ ] the mass of the sterile neutrino is deduced from the Hubble cooling of these particles in the primordial Universe and the fit in Section 2 . Some final comments are given also in this section. 


\section{The Self-Gravitating model}

We propose a model for the evolution of galactic halos of Vlasov particles (which interacts only gravitationally) in the static field of a spherical core of baryonic matter. The evolution of the distribution of the Vlasov particles must take into account not only the gravitational field of the baryonic core but also their own gravitational field. The statistical properties of self-gravitating systems are still not completely elucidated although this area is the object of continuous research since the sixties of the past century [1], [Q], 201, 20]].

Our model for the halo is based upon the following assumptions:

(i) The galactic core is represented by sphere with radius $R_{c}$ which contains almost all the baryonic mass.

(ii) DM was initially uniformly distributed inside the core. This could be unrealistic but our objective is not to describe the detailed evolution of the halo but merely to show that it is the stable asymptotic state for any perturbation.

(iii) The angular distribution of the velocities of the Vlasov particles is homogeneous in the unit sphere.

(iv) We consider two distributions for the velocity modulus: a Dirac delta and a more realistic one corresponding to a truncated parabola:

$$
f(v)=\left\{\begin{aligned}
\frac{81}{64} \frac{v^{2}}{\bar{v}^{3}} & \text { if } v<4 \bar{v} / 3 \\
0 & \text { if } v>4 \bar{v} / 3
\end{aligned}\right.
$$

where cut-off is chosen to verify the normalization condition and $\bar{v}$ is the average velocity. Sterile neutrinos produced by the resonant conversion of active neutrinos are supposed to end up with a strongly non-Maxwellian energy spectrum of this kind in the protogalaxies [ㄷ]].

Simulations of self-gravitating systems of particles constitutes a whole field onto itself. The $\mathrm{N}$-body problem is computationally very costly although a series of more and more efficient computer algorithms have been developed throughout the last forty years [22]. The original focus of these codes was the study of the evolution of globular cluster of stars [एष] and the formation of star binaries near the center of these objects is a major problem for these algorithms so regularizations schemes have also been developed to overcome this difficulty.

We expect that particles in the DM halo will escape from the baryonic core so close encounters among them should be rare. Moreover, elementary particles clouds are very different from globular clusters of stars. In order to perform simulations in a single modest computer we have used a mean-field gravitational model in which the gravitational field is derived from Gauss's theorem (by assuming that the DM particle distribution keeps spherically symmetric).

Another simplification in this problem is that the static baryonic core sets a natural scale for the positions, velocities and accelerations:

- Distances are measured in units of $R_{c}$, i. e., the radius of the core. 


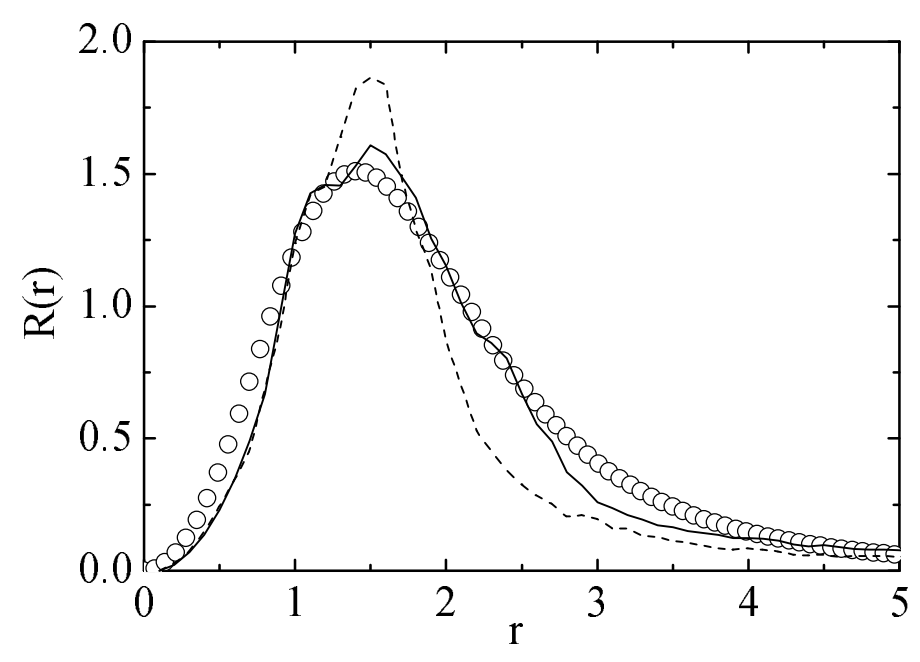

Figure 1: Simulation results for the radial distribution of DM in the galactic halo for $\bar{v}=1.2$ and a halo mass three times larger than that of the baryonic core, $M_{H}=3$ (dotted line) and $\bar{v}=1.3, M_{H}=4$ (solid line). Circles correspond to a Padé approximant for the density of the Milky Way halo derived from Kalberla \& Kerp model [[2] $]$. The radial distance is measured in units of $R_{c}=10 \mathrm{Kpc}$. We used 500000 time steps $(t=50)$ and $10^{5}$ particles.

- Our unit of mass is $M_{c}$, i. e., the mass of the baryonic core.

- Velocities are measured in units of $V_{c}=\sqrt{2 G M_{c} / R_{c}}$.

- Consequently, the unit of time is $R_{c} / V_{c}$.

- $V_{c}^{2} / R_{c}$ is the unit of acceleration.

By performing a standard Euler integration of Newton's equations for the DM particles's po-

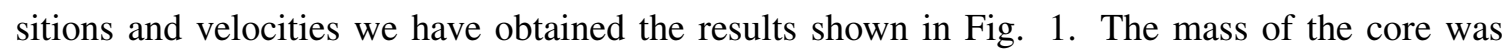
$M_{c}=75 \times 10^{9}$ solar masses. A nice agreement with Kalberla \& Kerp model [[23] for the distribution of mass in the DM halo is found. The halo distribution is asymptotically reached after 500000 time steps $(t=50)$ which roughly corresponds to 2 billion years.

Many observations seem to indicate an approximately constant dark matter density in the inner part of galaxies [24]]. This implies a quadratic power-law for $R(r)$ at short distances from the galactic center as shown in Fig. W. However, there are some cases, such as M31, where the evidence for a flattened dark matter halo at the galactic core [ [25], or instead a cuspy core [[26], is still the subject of a debate in observational astronomy. In the last case, our model is still useful but the results for the estimation of the DM particle mass should be different and this could change the preferred candidate for DM.

\section{The mass of the sterile neutrinos and final comments}

From the results in Sec. $\square$ we can estimate the average velocity of primordial sterile neutrinos at the time of formation of protogalaxies, $\bar{V}=1.3 V_{c}=330 \mathrm{~km} \mathrm{~s}^{-1}$. We must point out that this typical velocity is smaller than the escape velocity from the initial condition of uniformly distributed 
$\mathrm{DM}\left(V_{e} \simeq 568 \mathrm{~km} \mathrm{~s}^{-1}\right.$, assuming and halo with a mass $M_{H}=4$ times larger that the baryonic mass in the galaxy, $M_{c}$ ). Moreover, the escape velocity from the center of the stationary distribution in

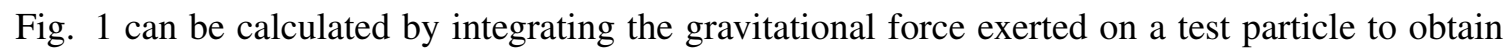
$V_{e} \simeq 382 \mathrm{~km} \mathrm{~s}^{-1}$. Consequently, after the reorganization of the halo into a stationary structure most of the primordial DM is held by the combined effects of the baryonic gravitational pull and the self-gravitation of the DM halo. This is confirmed by the best fit in Fig. $\square$ corresponding to a ratio $M_{H} / M_{c}=4$. This ratio is consistent with the relative abundance of DM and baryonic matter in a cosmological scale.

In our scenario sterile neutrinos decrease their momenta as a consequence of the expansion of the Universe just after their decoupling from the rest of matter. Being so weakly interacting this decoupling surely took place before that of the rest of the matter and radiation. Perhaps, even at a temperature of hundreds of $\mathrm{MeV}$ (for WIMPs a decoupling temperature of $k_{B} T \approx 1 \mathrm{MeV}$ is usually assumed).

In the very early Universe, the energy of a sterile neutrino is given by

$$
E=\mu c^{2}+\frac{k_{B} T}{a^{\star}}
$$

where $a^{\star}$ is the cosmological radius in term of the present radius, $\mu$ is the rest mass of sterile neutrinos and $T=2.7277 \mathrm{~K}$ is the Cosmic Microwave Background temperature also at the present. Linear momentum is then given by

$$
p^{2}=\frac{k_{B} T}{a^{\star}}\left[2 \mu+\frac{k_{B} T}{a^{\star} c^{2}}\right]
$$

Taking into account that linear momentum decreases as $1 / a(t)$ after decoupling we can calculate the kinetic energy of a DM particle at time $t$, that decouples at an earlier time $t^{\prime}$, as follows:

$$
Q\left(t, t^{\prime}\right)=\sqrt{\mu^{2} c^{4}+\left[\frac{a^{\star}\left(t^{\prime}\right)}{a^{\star}(t)}\right]^{2} \varepsilon_{\mathrm{rad}}\left(t^{\prime}\right)\left[\varepsilon_{\mathrm{rad}}\left(t^{\prime}\right)+2 \mu c^{2}\right]}-\mu c^{2},
$$

where $\varepsilon_{\text {rad }}=\varepsilon_{0} / a^{\star}(t)$ and $\varepsilon_{0}=2.35046 \times 10^{-4} \mathrm{eV}$ is the average energy of a CMB photon in the present day Planck spectrum. In Eq. 3.3 we assume that DM particles cool by the effect of Hubble expansion. Cosmological parameters have been determined with unprecedented precision by the WMAP project [ $[\mathbf{W}]$. In particular, we have used a flat cosmology with a Hubble constant $H=73 \mathrm{~km} \mathrm{~s}^{-1} \mathrm{Mpc}^{-1}$ and the following percentages of dark energy, dark matter and baryonic matter: $\Omega_{\Lambda}=0.72, \Omega_{\mathrm{DM}}=0.23$ and $\Omega_{\mathrm{B}}=0.05$. From this model we infer a mass of $3 \mathrm{keV}$ for the DM particles. This is consistent with a recent lower bound $m_{\mathrm{RP}}=2 \mathrm{keV}$ for the resonance production mechanism and $m_{\mathrm{NPR}}=1.77 \mathrm{keV}$ for the non-resonant mixing with active neutrinos obtained from a phase-space analysis of DM distribution in dwarf spheroidal galaxies [27].

We hope that this work should encourage specialists in structure formation in the early Universe to perform more global and detailed simulations replacing WIMPs by sterile neutrinos to find further support for the viability of this alternative hypothesis. 


\section{References}

[1] M. Milgrom, A modification of the Newtonian dynamics as a possible alternative to the hidden mass hypothesis, The Astrophysical Journal 270 pp. 365 (1983); A modification of the Newtonian dynamics - Implications for galaxies, The Astrophysical Journal 270 pp. 371 (1983).

[2] M. Kuhlen et al., The Via Lactea INCITE simulation: galactic dark matter substructure at high resolution ,J. Phys. Conf. Ser. 125:012008 (2008), [arXiv: 0810.3614].

[3] L. Acedo, A WDM model for the evolution of galactic halos, JCAP 07(2009)037.

[4] F. Zwicky, Die Rotverschiebung von extragalaktischen Nebeln, Helvetica Physica Acta 6 pp.110 (1933).

[5] S. M. Faber and J. S. Gallagher, Masses and mass-to-light ratios of galaxies, Ann. Rev. Astron. Astrophys. 17 pp. 135 (1979).

[6] V. C. Rubin, W. K. Ford and N. Thonnard, Rotational properties of 21 Sc galaxies with a large range of luminosities and radii, from NGC $4605(R=4 \mathrm{kpc})$ to $U G C 2885(R=122 \mathrm{kpc})$, The Astrophysical Journal 238 pp. 471 (1980).

[7] J. F. Navarro, C. S. Frenk and S. D. M. White, A Universal Density Profile from Hierarchical Clustering, The Astrophysical Journal 490 pp. 493-508 (1997).

[8] G. Gilmore et al., [arXiv:0804.1919v1].

[9] W. J. G. de Blok and A. Bosma, High-resolution rotation curves of low surface brightness galaxies, Astron. Astrophys. 385 pp. 816 (2002).

[10] J. A. Tyson, G. P. Kochanski and I. P. dell'Antonio, Detailed Mass Map of CL $0024+1654$ from Strong Lensing, The Astrophysical Journal 498 pp. L107 (1998), [astro-ph: 9801193 ].

[11] WMAP collaboration, D. N. Spergel et al., Three-Year Wilkinson Microwave Anisotropy Probe (WMAP) Observations: Implications for Cosmology, The Astrophysical Journal 170 pp. 377 (2007) [astroph: 0603449].

[12] Boomerang collaboration, P. de Bernardis et al., A flat Universe from high-resolution maps of the cosmic microwave background radiation, Nature 404 pp. 955 (2002) [astroph: 0004404 ].

[13] A. Dekel, J. Devor and I. Arad, Galactic Halo Cusp versus Core: Tidal Effects in Mergers in A New Era In Cosmology ASP Conference Series, (2002) T. Shanks \& N. Metcalfe Eds. [arXiv: astroph0201222v1].

[14] S. Dodelson and L. M. Widrow, Sterile neutrinos as dark matter,Physical Review Letters 72(1) pp. 17 (1994),[hep-ph: 9303287$]$.

[15] X. Shi and G. M. Fuller, New Dark Matter Candidate: Nonthermal Sterile Neutrinos,Physical Review Letters 82 pp. 2832 (1999), [astro-ph: 9810076 ].

[16] M. Shaposhnikov and I. Tkachev, The vMSM, inflation, and dark matter, Physics Letters B 639 pp. 414 (2006), [hep-ph:0604236].

[17] P. L. Biermann and A. Kusenko, Relic keV sterile neutrinos and reionization,Phsysical Review Letters 96091301 (2006), [astro-ph: 0601004 ].

[18] S. J. Aarseth, Dynamical evolution of clusters of galaxies, I, Mon. Not. R. Astron. Soc. 126 pp. 223 (1963). 
[19] M. Hénon, Sur l'évolution dynamique des amas globulaires, Annales d'Astrophysique 24 pp. 369 (1961); Sur l'évolution dynamique des amas globulaires. II.-amas isolé, Annales d'Astrophysique 28 pp. 62 (1965).

[20] D. Lynden-Bell, Negative specific heat in astronomy, physics and chemistry, Physica A 263 pp. 293 (1999), [cond-mat : 9812172$]$.

[21] W. E. Thirring, Selected Papers of W. E. Thirring with Commentaries, American Mathematical Society, 1997.

[22] S.. J. Aarseth, Gravitational N-Body Simulations, Cambridge University Press, 2003.

[23] P. M. W. Kalberla and J. Kerp, Hydrostatic equilibrium conditions in the galactic halo, Astronomy and Astrophysics 339 pp. 745 (1998).

[24] W. J. G. de Blok,The core-cusp problem, Advances in Astronomy Volume 2010, ID 789293 (2010).

[25] A. Banerjee and C. J. Jog, The flattened Dark Matter Halo of M31 as deduced form the observed HI scale heights, The Astrophysical Journal 685 pp. 254-260 (2008).

[26] E. Tempel, A. Tammm and P. Tenjes, Visibel and dark matter in M31-II. A dynamical model and dark matter density distribution, [arXiv:0707.4374v2].

[27] A. Boyarsky, O. Ruchayskiy and D. Iakubovskyi, A lower bound on the mass of dark matter particles, JCAP 03(2009)005, [arXiv:0808.3902]. 